\title{
Corela
}

Cognition, représentation, langage

14-1 | 2016

Vol. $14, n^{\circ} 1$

\section{La confidence théâtralisée dans les élégies de Marceline Desbordes-Valmore : le cas d'une écriture de l'hybridation générique, entre poésie et théâtre}

\section{Elena Thuault}

\section{(2) OpenEdition}

\section{Journals}

Édition électronique

URL : http://journals.openedition.org/corela/4576

DOI : $10.4000 /$ corela.4576

ISSN : 1638-573X

Éditeur

Cercle linguistique du Centre et de l'Ouest - CerLICO

Référence électronique

Elena Thuault, « La confidence théâtralisée dans les élégies de Marceline Desbordes-Valmore : le cas d'une écriture de l'hybridation générique, entre poésie et théâtre », Corela [En ligne], 14-1 | 2016, mis en ligne le 27 juin 2016, consulté le 19 avril 2019. URL : http://journals.openedition.org/corela/4576 ; DOI : 10.4000/corela.4576

Ce document a été généré automatiquement le 19 avril 2019

\section{(†) (2)}

Corela - cognition, représentation, langage est mis à disposition selon les termes de la licence Creative Commons Attribution - Pas d'Utilisation Commerciale - Partage dans les Mêmes Conditions 4.0 International. 
La confidence théâtralisée dans les élégies de Marceline DesbordesValmore : le cas d'une écriture de l'hybridation générique, entre poésie et théâtre

\author{
Elena Thuault
}

Mes remerciements à Madame Nathalie Garric pour ses relectures bienveillantes ${ }^{1}$

\title{
Introduction
}

(1) Marceline Desbordes-Valmore (1786 - 1859) fut une femme de lettres considérée comme ayant appartenu au pré-romantisme et au romantisme français. Elle écrivit un roman, des contes et surtout de la poésie lyrique. Elle s'illustra principalement dans le genre élégiaque qui traversa toute sa vie littéraire, de ses premiers poèmes parus vers 1814 dans des keepsakes et revues féminines, jusqu'à ses derniers, endeuillés et publiés posthumes en 1860. Elle fut aussi comédienne de profession, et bien qu'elle n'écrivitt pas de théâtre, elle conserva un goût prononcé pour celui-ci. Elle en connaissait les spécificités d'écriture qu'elle intégra dans ses élégies amoureuses, ainsi marquées par une hybridité générique.

3 (2) Au cœur de cette réflexion sur le genre poétique, marqué par des conventions scripturales stables et spécifiques de nature typographique ou stylistique (vers, rimes, figure de style, fonction expressive, etc) mises au service de la forme et du rythme, l'expression d'hybridité générique nécessite une clarification sémantique. Dion, Fortier et Haghbaert la définissent comme une " combinaison de plusieurs traits génériques 
hétérogènes mais reconnaissables, hiérarchisés ou non, en un même texte » (2001 : 353). Cette définition, bien qu' intéressante, suscite un embarras car elle pourrait correspondre aussi à celle de l'inter-généricité, sur laquelle nous reviendrons ultérieurement, et dont elle ne semble pas être dissociée. Il y aurait pourtant une différence entre hybridité et intergénéricité, tout comme entre hybridité et hybridation en littérature. L'hybridation générique, considérée comme un "processus » (Mihajlovska,2012 : 7), est " un geste créateur [...] [qui] ouvre deux sèmes principaux, [...] celui du croisement [...] et [...] celui de l'hétérogène »( Baby, $2005:$ 6). Nous redéfinissons l'hybridation générique comme un procédé scriptural qui prélève des éléments caractéristiques d'un genre A et les implante dans un genre B. La notion de processus de transplantation et de croisement y est essentielle et permet d'opérer une distinction avec l'hybridité. L'hybridation insiste sur la notion d'action, de mobilité et d'évolution, avec « le suffixe en -tion dénotant en français polysémiquement l'acte et le produit de l'acte » (Kerbrat-Orecchioni 2009 : 33), tandis que l'hybridité, par son suffixe, prend sens dans le figement, dans l'immobilité (Baby 2005 : 6). Cette dernière est alors à envisager comme l'état résultatif et statique de la première. L'hybridation pose évidemment la question des frontières génériques, envisagées dans leur décloisonnement, dans leur dépassement, et ce à travers deux phénomènes proches mais distincts : la trans-généricité et l'inter-généricité, avec ou sans tiret. La transgénéricité, conformément au sens du préfixe trans, correspondrait selon nous à la migration d'un genre A vers un genre B qui épouserait partiellement ou intégralement les caractéristiques de l'autre jusqu'à perdre les siennes. Dans le cas d'une transgénéricité partielle, une inter-généricité s'observerait alors, avec une définition proche de celle de Dion, Fortier et Haghbaert pour l'hybridité. L'intergénéricité serait une relation de co-présence dans un même texte de plusieurs traits relevant de genres différents. Toujours selon nous, il est possible de constituer une chaîne d'étapes conceptuelles en posant qu'il y a un processus d' hybridation avec des phénomènes de transgénéricité ou d'intergénéricité qui aboutissent à un état d'hybridité. Après cette digression sur ces nécessités théoriques et terminologiques, nous en revenons à Marceline Desbordes-Valmore et aux éléments d'hybridation dans ses poèmes. Quels sont les codes et artifices divers qu'elle transplante du théâtre vers la poésie, et qu' apportent-ils à ses élégies sentimentales?

(3) Un premier et second axes seront consacrés à l'étude d' une scénographie de la confidence et d'une figure symbolique de confidente, qui, comme dans le genre théâtral, sont présentes dans ces poèmes. Dans un troisième point, nous examinerons l'écriture dramaturgique en elle-même qui a été adoptée et qui théâtralise cette confidence. Dans une perspective interdisciplinaire entre littérature et linguistique, la confidence sera abordée sous l'angle des théories stylistiques, sémantiques et pragmatico-énonciatives de l'analyse du discours de tradition française (ADF).

\section{Une scénographie littéraire de la confidence}

(4) Si le terme confidence n'est jamais employé dans les poèmes, celle-ci est pourtant présente sémantiquement et thématiquement, et ce par l'élaboration d'un lexique spécifique et par l'inclusion d'un destinataire jouant le rôle de figure de confidente. 


\subsection{Sémantisme et isotopie de la confidence}

8 (5) Un faisceau de sèmes est associé à la confidence et permet de considérer qu'il y a bien un acte de se confier. Pour démontrer cette hypothèse d'une présence in absentia, nous rappellerons dans un premier temps la définition de la confidence, puis nous verrons comment, à partir de cette définition, le lexique fait naître une isotopie de la confidence.

10 (6) La confidence est définie comme une déclaration faite en secret à quelqu'un ${ }^{2}$, et admet pour expressions synonymiques dire un secret ou épancher son coeur. Les définitions des dictionnaires ${ }^{3}$ font toutes apparaitre explicitement deux sèmes récurrents, celui de communication et celui de secret. Ils composent ce qui serait l'essence de la confidence, que nous schématisons sous la forme d'un noyau sémantique, inspiré des travaux de la Sémantique des Possibles Argumentatifs (SPA) de Galatanu (depuis 1999) : CONFIDENCE = COMMUNICATION + SECRET. Ce noyau autorise à redéfinir la confidence telle une communication secrète. Parler de communication signifie qu'il y a un émetteur, un récepteur, un message/un dictum, avec un mode de transmission particulier, qui serait le secret. Le support de la communication peut aussi jouer un rôle, écrit ou oral. Le genre lui-même peut être une extension de ce support.

(7) Conformément à cette définition, les élégies de Marceline Desbordes-Valmore mettent bien en scène un émetteur, appelé sujet lyrique ou je-lyrique en poésie, avec ou sans tiret, qui s'adresse à un récepteur explicite, avec un objet du dire et un mode de transmission. Le poème « Révélation » déploie ce noyau de la confidence :

Oh! Ne me dis jamais qu'il faudra se guérir ;

Qu'aimer use le cœur et que tout doit mourir ;

Car tu me vois dans l'âme : approche, tu peux lire ;

Voilà notre secret : est-ce mal de le dire?

Vois-tu, d'un cœur de femme il faut avoir pitié !4

Le support choisi est l'écrit, « tu peux lire », mais avec la mise en abyme d'une oralité, par la répétition du verbe dire, créant une tension de va-et-vient entre l'écrit et l'oral, à l'image du genre théâtral. Revenons sur ce noyau élaboré à partir de deux éléments. Ceux-ci peuvent aussi s'apparenter à ce qui serait, selon Rastier dans sa Sémantique interprétative (1987), une molécule sémique, c'est à dire l'union de deux sèmes au minimum. La notion de molécule nous intéresse, car actualisée au moins deux fois dans un texte, elle créerait une isotopie, soit " au sens strict l'itération d'un sème d'une lexie à l'autre [ou] au sens large, une itération sémantique quelconque " (Stolz $2006: 100-101)$. Chez Desbordes-Valmore, la composition d'une molécule peut varier d'un poème à l'autre, mais nous considérons qu'il faut qu'elle soit constituée de plusieurs sèmes du sémème de la communication $^{5}$, toujours couplé avec le sème du secret. Dans l'extrait précédemment cité, la molécule se composerait de 5 éléments : 4 sèmes du sémème de la communication (l'énonciateur : je - le récepteur : tu - le message : cœur/âme/coeur de femme - le support de la communication : dire/lire) auxquels s'additionne celui du secret. Nous n'estimons par contre pas nécessaire la duplication de la molécule. Sa richesse en elle-même suffit, surtout lorsque ses sèmes sont disséminés dans une large portion textuelle, touchant ainsi à la macrostructure de l'écrit. 


\subsection{L'inscription discursive d'une figure de confidente}

14 (8) Un personnage féminin est donc inclus explicitement dans le rôle du récepteur, indispensable dans cette communication secrète. Si l'on relie ce destinataire au contexte de la confidence construit par l'isotopie, le lecteur pourra considérer que ce destinataire fonctionne comme une figure de confidente. La confidente, rappelons-le, est un personnage rencontré dans le genre théâtral. Elle en est même un artifice ayant pour fonction de provoquer la parole d'un personnage important, c'est à dire de permettre au spectateur d'en connaître l'intériorité. Nous développerons ultérieurement les caractéristiques de la confidente. A ce stade, seuls nous intéressent ses modes d'apparition dans le discours, au nombre de trois.

(9) Le premier cas d'inscription discursive s'opère par la présence d' un syntagme " ma sœur », en position d'adresse lyrique, à intégrer dans les procédés de l'interpellation : « Ma sœur, il ne faut me blâmer $»^{6}$. Nous avons identifié les occurrences de cette adresse dans une dizaine d'élégies d'inspiration amoureuse. L'emploi du possessif suggère de prime abord un sentiment de proximité affective, important, car il serait une condition nécessaire et suffisante pour qu'il y ait acte de confidence. Le possessif et le terme de parenté construisent surtout une désignation référentielle spécifique, non absolue mais co-textuelle, en lien avec l'énonciateur dont cet autre semble dépendre pour exister. Au moins neuf poèmes ${ }^{7}$ contiennent ce syntagme en position $\mathrm{d}^{\prime}$ adresse lyrique incluse dans le tissu textuel. Deux d'entre eux sont aussi intitulés « A ma sœur » : « Ma sœur, j'ai vu la mort à la triste lumière $\|^{8}$. Les occurrences de ce syntagme sont toujours identiques et tissent une auto-intertextualité entre ces textes, écrits pour la plupart entre 1815 et 1825, à l'époque où Desbordes-Valmore vivait confortablement de sa carrière de comédienne, avec une culture théâtrale érudite. Et ces poèmes ont bien un lien explicite avec le théâtre car ce syntagme rappelle un passage d'une pièce du dramaturge Corneille dont elle avait souvent interprété des œuvres. Il s'agit d' Agésilas, qui s'ouvre par cette même adresse lyrique :

\section{AGLATIDE}

Ma sœur, depuis un mois nous voilà dans Ephèse,

Prêtes à recevoir ces illustres époux

Que Lysander, mon père, $a$ su choisir pour nous

(Agésilas, acte I, scène 1, vers 1 à 3.)

Dans cette scène, les soeurs Aglatide et Elpicine s'entretiennent de leurs futurs époux et avouent souffrir de ces mariages arrangés. L'adresse lyrique reprise par DesbordesValmore est alors une allusion textuelle, opérant des « rappels mémoriels[...] à des dires antérieurs » " (Moirand 2007 : 12). Elle repose ici sur trois points : un syntagme, un lien de sororité et une scène de confidence. Relevant du dialogisme interdiscursif, cette allusion inscrit les mots d'un autre auteur dans le discours et élabore une hétérogénéité énonciative mise en lumière par Authier-Revuz (1984) reprenant les travaux sur le dialogisme de Bakhtine. Cette hétérogénéité est constitutive dans ces élégies, c'est à dire dissimulée clandestinement dans le discours, et repose sur les trois éléments cités qui donnent à constater un phénomène d'hybridation, puisqu'ils appartenaient à l'origine au genre théâtral avant d'être implantés dans ces poèmes. Une autre forme d'hétérogénéité apparaît alors, non plus énonciative mais générique.

(10) Dans le deuxième cas d'actualisation, la confidente demeure une figure de l'altérité maintenue dans une indétermination identitaire, sans référence intra ou extradiscursive : 
Un jour, écoute..., un jour j'étais bien malheureuse !

[...] tes regards émus, sur les miens attachés,

Relisent nos secrets dans nos âmes chéries.

\section{(Siblot 1997 1998, Moirand 2003). Elle renvoie au titre d'un recueil éponyme de Maurice} Scève ${ }^{12}$ : Délie, Objet de plus haute vertu. Ce fut le premier recueil de poésie amoureuse écrit à la manière de Pétrarque en France et publié en 1544. Avec Desbordes-Valmore, la figure de Délie n'est plus celle que l'on aime, mais celle à qui l'on se confie telle une aînée ayant l'expérience de l'amour. Bien que la figure du sujet lyrique maintienne une distance respectueuse, une subtile image de sororité est esquissée par le parallélisme de deux féminités souffrant à travers les siècles, et personnifiant habilement deux inspirations poétiques à travers l'histoire littéraire. Notons aussi promptement que la figure de Délie, avant Marceline Desbordes-Valmore, fut réactualisée par deux autres écrivaines : Madame de Genlis, dans un de ses romans, et Victoire Babois, dans un poème, où Délie et une amie discutent dans une scène de confidence avec des échanges verbaux, comme au théâtre. Ce poème hybride a aussi inspiré Marceline Desbordes-Valmore. Ce dialogisme interdiscursif tissé avec la figure de Délie lui permit d'ancrer symboliquement sa poésie dans la tradition d'une littérature amoureuse pétrarquiste, élégante et cultivée, ainsi que de se positionner dans un autre héritage, féminin, indispensable à l'époque pour exister en tant que femme auteur.

\subsection{L'élaboration d'une scénographie littéraire de la confidence}

(12) Les itérations sémantiques et la figure littéraire co-construisent une scénographie de la confidence. Nous citons intégralement la définition que Garric et Calas en donnent car elle permettra d'appréhender le fonctionnement de la scénographie valmorienne :

Mise en scène interne avec ses propres acteurs linguistiques, sa propre topographie et sa propre temporalité d'un discours. La scénographie est construite par 
l'énonciation et déterminée par le genre de discours, elle est comparable à la scène de théâtre qui propose une représentation scénique ». (2007:191). générique pouvant inclure la confidence dans ses conventions, ce qui est possible pour le genre poétique et pour le genre théâtral. A l'époque où écrivait Marceline DesbordesValmore, il était admis que l'entité qui dit je en poésie épanche les élans de son cœur, la poésie lyrique étant associée depuis Aristote et sa Poétique à l'expression d'une intimité dévoilée. Pour le genre théâtral, le fil narratif contient des scènes de communications secrètes : de la confidence de personnages amoureux et malheureux, aux histoires de complots tramés. Poésie lyrique et théâtre intègrent donc chacun dans leur essence la dualité du caché et du montré qui caractérise la confidence dans l'art. Ainsi, la confidence qui est un point commun aux deux genres, est devenu pour la poétesse un point d'interaction, un vecteur, qui offre la possibilité de circuler de l'un à l'autre.

La scénographie valmorienne ne repose pas uniquement sur le dire d'une confidence, dans une valeur performative. Des éléments spécifiques viennent l'agrémenter, la renforcer, avec une écriture narrativo-descriptive, répondant plus ou moins aux critères du récit selon Brémond (1973) qui reprit, dans une postulat structuraliste, les travaux de Propp sur les contes russes. Il y a tout d'abord des verbes d'actions qui déroulent un fil narratif, créant une temporalité ordonnée, évoquée dans la définition citée. Ce fil permet l'élaboration de scenarii, avec l'exemple d'une femme qui s'adresse à sa sœur pour lui demander de sauver son ancien amant qu'elle aime encore et dont la vie est à présent en danger :

Qu'ai-je appris ! Le sais-tu ? Sa vie est menacée, [...]

J'ai couru... je suis faible... et ma langue glacée

Peut à peine... Ma sœur, je l'aime donc toujours ! ${ }^{13}$

En complémentarité de ces scenarii, des lieux sont intégrés à la scène d'énonciation, avec des itinéraires parcourus ou à parcourir qui tissent une topographie :

Quoi! Les flots sont calmés, et les vents sans colère

Aplanissent la route où je vais m'égarer!

J'ai vu briller le phare et l'onde qui s'éclaire. ${ }^{14}$

Les indications spatiales, les flots, la route, le phare, l'onde, se trouvent subordonnées non pas à des états, comme cela serait attendu en poésie, mais à des actions, où je vais m'égarer, comme dans l'écriture dramaturgique qui doit planter des décors aux actions. Les scenarii, l'élaboration d'une spatialité avec un phénomène d'intégration d'indications locatives dans les vers, et la subordination-même de la topographie aux actions, sont aussi trois procédés d'hybridation génériques empruntés au théâtre.

26 (14) Enfin, concernant l'expansion de la scénographie, trois mouvements sont observables. Le premier mouvement a son point d'origine à partir de la figure de la confidente, à partir de cette adresse lyrique sur laquelle le vers s'élabore. Ses occurrences sont souvent en double position initiale, dans le premier hémistiche du premier vers qui ouvre le poème :

T 1 T2 T3 T4

0102

Ma sœur, / il est parti ! // Ma sœur, / il m'abandonne !

Dans cet extrait, l'adresse lyrique est mise en valeur rythmiquement par le port de l'accent tonique (noté $\mathrm{T}$ ) qui coïncide avec l'accent oratoire ${ }^{15}$ utilisé dans les textes ayant 
pour fonction d'être lu ou déclamé publiquement (noté 0 ). Par un phénomène d'itération, elle se fait anaphore rhétorique en scandant chaque début d'hémistiche. Se créée alors un double rythme binaire enchâssé dans le rythme binaire principal qui scinde le vers en deux. L'anaphore rhétorique possède un rôle architectural, tant grammatical que rythmique. Elle impulse et structure le vers, et permet alors à la figure de confidente d'initier et de structurer la scénographie. La deuxième possibilité repose sur le mouvement inverse, donnant la primauté au sémantisme, avec une ou des molécule(s) sémique(s) préexistant au destinataire, surtout lorsque celui-ci reste anonyme dans la suite du texte :

Un jour, écoute... un jour j'étais bien malheureuse !

Dans ce vers initial, le verbe " écoute ", placé dès le premier hémistiche, introduit l'acte de confidence placé au premier plan par rapport à l'allocutaire, anonyme et secondaire, et n'ayant d'existence que par la deuxième personne du singulier qui fléchit la forme verbale. Enfin, le troisième cas construit un équilibre entre itérations sémantiques et figure de confidente dans cette relation de co-présence, et se retrouve dans le cycle de Délie. Après la question de l'inscription discursive de ces figures de confidentes unies autour d'un motif explicite ou implicite de sororité, il convient d'en étudier les représentations.

\section{La confidente, une figure aux identités plurielles et symboliques}

(15) Notre analyse fera apparaître un cheminement identitaire, de la sœur réelle à la sœur sémiotique (ou fictive), de la sœur sémiotique à la sœur d'élection, la lectrice, et de la lectrice à la figure auctoriale.

\subsection{De la sœur empirique à la sœur sémiotique}

30

31

(16) La figure de la sour devient une stratégie discursive intéressante : rien que par le syntagme qui la désigne, elle instaure un lien entre la scène de communication, celle de l'être de chair qui écrit des poèmes, et la scène d'énonciation, celle du sujet lyrique, un être de discours, un être de papier. Ce lien va jusqu'à créer une confusion entre ces scènes, où le lecteur pourrait supposer que le sujet lyrique correspondrait à l'auteure qui avait deux sœurs, Cécile et Albertine, et qui aurait pu confier par écrit ce qu'elle ne pouvait dévoiler de vive voix. Ce brouillage énonciatif fonctionne comme un effet de réel qui répand l'illusion d'une écriture autobiographique, où l'écrivain, le sujet lyrique et la figure mise en scène renverraient tous trois à une même entité, avec un principe de sincérité assurant la cohésion entre eux, comme développé par Philippe Lejeune depuis son Pacte autobiographique (1975). Bien sûr la recherche de l'illusion du vrai n'est pas nouvelle dans l'élégie. Elle se rencontrait déjà chez Ronsard et du Bellay. Quant à l'inclusion de faits autobiographiques, André Chénier (1762-1794), avec la retranscription de son emprisonnement et de ses derniers jours sous la Terreur, ou Victoire Babois (1760-1839), avec l'écriture de ses Elégies maternelles suite au décès de son enfant, en donnent le coup d'envoi avec des poésies publiées la même année, en 1805, bien qu'écrites plus d'une décennie auparavant. Desbordes-Valmore s'est inscrite dans une mouvance où 
la poésie lyrique et les attentes autour de celle-ci étaient en évolution au début du XIXe siècle, avec une primauté accordée à la sincérité autobiographique, ou du moins à ce qui pouvait le plus possible s'en rapprocher. Il s'agissait de faire sincère, de faire vrai, comme le prônait Madame de Staël dans son De l'Allemagne, écrit en 1810 et publié en 1813, prenant pour modèle le lyrisme de Goethe et de ses confrères déjà en vigueur dans les pays germanophones :

Le véritable poëte conçoit, pour ainsi dire, tout son poëme [...] au fond de son âme ; sans les difficultés du langage, il improviserait, comme la sibylle et les prophètes, les hymnes saints du génie. (Madame de Staël, De l'Allemagne) ${ }^{16}$

Le terme de " poëte » employé par Madame de Staël n'est pas à entendre comme un renvoi référentiel au sujet empirique, mais désignerait une posture à adopter vis à vis de son œuvre. La notion d'intériorisation fait ressortir la dimension humaine du créateur et le pose en homme - Homme, c'est à dire à la fois en sujet affectif et en sujet philosophique, avec une sensibilité à assumer pour atteindre une subjectivité convaincante. Pour se faire, il faut tisser des émotions à pouvoir mettre en mots de manière crédible, c'est à dire semblant avoir été intimement vécues par l'artiste, quand bien même celui-ci aurait mené une vie fort différente. Le motif de la confidence qui a nourri les premières poésies de Marceline Desbordes-Valmore semble alors s'être vraiment inscrit dans le contexte d'émergence du Romantisme, où l'énonciation littéraire se fit le miroir d'un contrat de parole inscrite dans une valeur de vérité, jouant sur l'illusion du vrai, pour replacer l'individualité et l'affectivité humaines au cœur des préoccupations scripturales.

(17) L'illusion référentielle et la superposition des plans n'est pas la seule force offerte par cette sœur - confidente qui mue en signe sémiotique. Vue à travers le prisme de la sémiotique, toute figure littéraire est un signe symbolique et iconique, c'est à dire un système de signes renvoyant à d'autres signes et à leurs fonctions, avec des valeurs symboliques et potentiellement emblématiques de toute sa catégorie. La confidente valmorienne possède une identité, avec un sexe, une relation spécifique avec l'énonciatrice, une fonction dramaturgique traditionnelle (cautionner le dire du sujet lyrique), et une fonction scénographique spécifique (le rôle architectural précédemment mis en lumière). Ce signe possède aussi une histoire inscrite dans une mémoire littéraire collective où l'ont précédé les autres figures de confidentes auxquelles il renvoie. Il écrit aussi sa propre histoire : il devient avec Desbordes-Valmore une nouvelle licence dans le discours poétique.

\subsection{De la sœur sémiotique à la sœur d'élection : la lectrice}

(18) Au théâtre, le spectateur est désigné par le terme de « tiers inclus » (Ubersfeld 1977), puisqu'il est intégré au procédé discursif de la double énonciation qui joue sur la superposition déjà évoquée du plan énonciatif, un personnage $\mathrm{x}$ s'adressant à un personnage $y$, et du plan communicatif, un dramaturge s'adressant à son spectateur. Nous défendons l'idée, peu reconnue, qu'un poète peut aussi manier la double énonciation pour s'adresser à son lecteur qui devient alors un tiers inclus, surtout dans le cas d'un flottement ou d'un effacement identitaire dans l'adresse :

Le lecteur-allocutaire est témoin non plus d'une adresse précisément orientée mais d'un flottement structurel de l'adresse [...]. Là où le dialogue se dénoue avec le ou la destinataire explicite, il se renoue obliquement avec une instance polyvalente anonyme, un « tiers inclus ». (Sermet, 1996 : 94). 

avec un TUd, le sujet destinataire, intradiscursif, et le TUi, un sujet interprétant, extradiscursif (Charaudeau, 1983 : 41). Ce postulat nous ramène aux théories de la réception et de la lecture élaborées par l'école de Constance dans les années 1970, avec surtout les travaux d' Iser qui se pencha sur le cas du lecteur explicite dans un texte, et qui défendit l'idée que le sens était toujours à construire par ce dernier. Chez DesbordesValmore, le TUd et un TUi, s'ils apparaissent dans le texte, sont toujours superposés, comme dans le poème « Révélation » qui ouvre Les Pleurs, avec le lecteur ou la lectrice qui devient le/la confident(e) dans le texte. Cette assimilation est permise par une autre fusion identitaire, celle du Je lyrique et de l'auteure, rendue possible par la fonction préfacielle du texte où traditionnellement un énonciateur renvoie à un écrivain qui expose ses intentions. Dans cette préface auctoriale versifiée, le sujet lyrique déclare :

Car tu me vois dans l'âme : approche, tu peux lire ${ }^{17}$,

et renvoie à la scène de communication en s'adressant explicitement au lecteur, à la lectrice. Le système de double énonciation permet de mettre en exergue cette lectrice confidente en le plaçant au coeur de ce procédé discursif. Ainsi, la confidente peut tout autant renvoyer à une sœur biologique, « Que de fois dans tes yeux j'ai reconnu ma mère $! »^{18}$, qu'à une figure intradiscursive et fictive, ou qu'à une lectrice réelle mais fictionalisée par le discours et dans le discours. Et tous les poèmes de confidence déploient une figure aux identités plurielles et triadiques, entre réalité et fiction, entre confidence et artifice poétique, entre corps littéraire et corps de chair lisant un livre, avec une esthétique non sans rappeler celle du théâtre baroque du 17e siècle reprenant les concepts du speculum mundi ${ }^{19}$ qui interrogeait le rapport que l'art entretenait avec le réel. La création était perçue comme un jeu de miroirs savamment disposés pour brouiller les références, avec le lecteur/spectateur toujours en position de destinataire suprême, comme dans L'illusion comique de Corneille, demeurée emblématique du baroque littéraire.

\subsection{De la lectrice à l'auteure, ou comment le tu permet au je de se construire}

(19) Tisser une intimité entre le je lyrique et sa destinataire offre la possibilité à Desbordes-Valmore de mettre en abyme une relation fantasmée avec sa lectrice qu'elle invite à accéder à sa part intime : ses précieux poèmes livrés aux yeux d'autrui. La confidence fonctionne ainsi comme un espace communautaire de partage émotionnel qui décloisonne les univers traditionnellement séparés de l'écrivain et du lecteur, ainsi rapprochés l'un de l'autre par la douleur universellement ressentie, comme dans « A celles qui pleurent ", autre poème à fonction préfacielle et métatextuelle qui ouvre cette fois le recueil Bouquets et Prières, en 1843 :

Vous surtout qui souffrez, je vous prends pour mes sœurs:

[...] Prisonnière en ce livre une âme est contenue :

Ouvrez, lisez : comptez les jours que j'ai soufferts :

Pleureuses de ce monde où je passe inconnue,

Rêvez sur cette cendre et trempez-y vos fers

[...] Qui donne son secret est plus tendre que folle :

Méprise-t-on l'oiseau qui répand sa chanson ? ${ }^{20}$.

Corela, 14-1 | 2016 
retrouve dans ce texte toute une isotopie de la confidence alliée au motif des larmes que l'on verse pour l'autre dans cette relation qui renforce la dimension psycho-affective de la confidence et qui permet au Je d'élaborer un ethos ${ }^{21}$ auctorial de femme sensible. D'origine socio-culturelle, l'ethos de sensibilité fut un enjeu primordial à l'époque de l'écrivaine. Il se développa dans les salons aristocratiques à partir de la seconde moitié du 18 e siècle, avec le culte des larmes comme expression d'un cœur noble et vertueux, avant de devenir en littérature un trait constitutif du génie préromantique et romantique, défini par la capacité d'un artiste à sentir et à agir (Vratskidou, 2011 : 44), soit à vibrer en harmonie avec ce qui l'environne, que cela soit la nature dans un sentiment de contemplation, ou ses semblables dans un sentiment d'union et de compassion. Chez Desbordes-Valmore, la sensibilité et l'empathie, qui développent donc le génie, sont le plus souvent déployées à partir de la confidence qui se travestit alors sous des traits polymorphes, à la fois matrice du dire et objet du dire, « donne son secret », « répand sa chanson ", et ce dans une boucle réflexive et métatextuelle, " ouvrez, lisez », ou " approche, tu peux lire ».

boucles réflexives du métalangage (Authiez-Revuz 1996) dans "Révélation » et " A celles qui pleurent ", activent une modalité autonymique en rapport avec la question de l'ethos. Elles invitent à considérer ces poèmes non plus seulement comme des poèmes préfaciels mais comme des arts poétiques où Desbordes-Valmore démontre son talent scriptural par sa capacité à faire perdre ses repères au lecteur. Le brouillage référentiel dans l'adresse/apostrophe, la pluralité identitaire de la confidente, l'illusion d'un épanchement entre autobiographie et fiction, entre sincérité lyrique et artifice théâtral, sont tous des éléments d'hybridation générique qui invitent le lectorat à repenser et à reconceptualiser la poésie par rapport au théâtre :

En repoussant les frontières de ses parties constitutives, le texte hybride bouscule donc également la vision du monde du lecteur. En effet, [...] en l'obligeant à plonger dans des univers protéiformes et à rechercher des points d'interaction, de réunion, entre des éléments divergents, l'œuvre hybride l'arrache au confort du monde qui lui est familier pour l'amener à reconsidérer ses certitudes et à étendre son champ de vision. (Mihajlovska 2012 : 16).

41 Avec ces deux poèmes, à la fois préfaces et arts poétiques offrant la démonstration d'une défrontiérisation générique, un autre ethos auctorial est esquissé en filigrane, celui d'une artiste - intellectuelle et d'une intellectuelle - artiste, à rebours de l'idée d'une créativité spontanée et émotive dans laquelle Desbordes-Valmore et les autres femmes artistes furent cantonnées et emprisonnées par des critiques, confrères ou lecteurs à la misogynie souvent débridée ${ }^{22}$, incapables qu'elles auraient été d'intellectualiser un art, régi seulement par l'imagination et les instincts lorsque celui-ci se décline au féminin. Une autre forme de défrontiérisation pourrait alors être à l'œuvre, invitant le lectorat de l'époque à étendre son champ de vision, plus seulement littéraire et générique, mais identitaire et sexué.

Soulignons, pour achever cette étude sur les ethê que ceux-ci ont une existence discursive différente, analysable à partir du couple notionnel ethos dit /ethos montré (Maingeneau, 2002). L'ethos de sensibilité est dit, car son inscription est intratextuelle et se fait à travers un champ lexical de l'émotivité et des larmes dont la présence est récurrente dans ce corpus. Pour l'ethos de l'artiste intellectuelle, la poétesse ne se pose pas comme telle. Cette présentation de soi ne s'élabore pas dans le texte mais est la résultante d'un certain discours. On la qualifiera alors de montrée, touchant à ce que l'on pourrait considérer 
comme du paradiscursif et non du discursif. Cet ethos apparait comme la conséquence de la capacité à écrire des arts poétiques. Tout poète écrivant des arts poétiques projetait consciemment une image de lui de théoricien capable d'intellectualiser son art et de prendre place dans les débats d'idées de son temps. Puisque cette posture était réservée aux hommes, Marceline Desbordes-Valmore dut recourir à des stratégies : composer des arts poétiques sur le mode du caché, du camouflé, c'est à dire sans que ceux-ci semblent en être, et surtout, dépasser la question même d'une réflexion en proposant simultanément une réflexion et une démonstration autour de la figure du poète sensible devant parler au cœur, et ce en donnant l'illusion d'une intimité du dire passant pas la confidence.

\section{De l'oralité à la théâtralisation de la confidence}

(22) Nous verrons dans ce dernier point comment le style de Desbordes-Valmore théâtralise doublement la confidence selon les deux sens que nous accordons au terme théâtralité : 1- tout ce qui a trait au théâtre et à l'écriture dramaturgique mais hors du genre théâtral, c'est à dire dans un autre genre. 2- ce qui fait naître des images visuelles permettant de se représenter mentalement les faits. Cette double théâtralité est élaborée par des marqueurs d'oralité, par la récurrence d'isotopies du mouvement, et enfin par une forte intertextualité qui confère aux poèmes un style de la tragédie.

\subsection{Marqueurs d'oralité et actes de langage}

(23) Dans le cas de Desbordes-Valmore, nous pouvons parler d'un véritable style de l'oralité, élaboré sur les modalités d'énonciation, avec les interjections conçues comme marqueurs de ces modalités, ainsi que sur la mimétique d'une parole essoufflée.

(24) Les modalités exclamatives et interrogatives retranscrivent graphiquement des variations prosodiques et intonatives (Maillochon \& Bassano 2003). Tous les poèmes du corpus contiennent des tournures exclamatives et interrogatives, avec une mention spéciale pour " A ma sœur / Que veux-tu ? Je l'aimais ", "A ma sœur / Qu'ai-je appris ! Le sais-tu ? ", s'ouvrant sur des interrogations. La modalité interrogative est la plus intéressante dans le cadre d'une analyse d'un discours poétique puisqu'elle y est traditionnellement rare, voire inexistante. Au sein de ces modalités exclamativointerrogatives, les marqueurs linguistiques d'oralité les plus fréquemment rencontrés dans ces élégies sont les interjections « ah! », « eh!» et " Quoi !? », soit « un segment d'énoncé [...] bref, comme hors structure et donc rythmiquement différent du reste de l'énoncé ». (Rochette $2007: 278$ ), avec une recherche de l'intégration de l'oralité dans l'écrit, problématique essentielle au genre théâtral. « L'interjection participe activement de cet effet de réel en mimant la spontanéité du locuteur qui s'exprime[...]. » (Rosier 2004 : 23). Cette " mimétique de l'oral» (idem) est aux antipodes des horizons attendus dans une élégie, genre poétique le plus noble et raffiné qui soit, devant proscrire le prosaïsme de l'oralité avec certaines interjections fonctionnant comme des accidents du langage ${ }^{23}$, comme des scories employées par les dramaturges classiques, et reprises par la poétesse, pour conférer un côté subjectif et spontané à un énoncé, et retranscrire « la mise en voix et en conscience des personnages » (Rosier $2006: 116)$.

(25) Ces modalités d'énonciation, qu'elles opèrent sur une phrase ou sur un segment bref d'énoncé, possèdent différentes fonctions et utilités langagières, et appartiennent à la 
catégorie des subjectivèmes qui donnent des informations sur " [1'] investissement de l'énonciateur et [sur l'] expression de la sensibilité » (Garric \& Calas 2007 : 74). Placées en position initiale du vers, les interjections donnent l'illusion que le sujet lyrique réagit et répond à un dire antérieur. L'auteure applique alors une règle importante en dramaturgie : respecter l'organisation préférentielle, c'est à dire assurer une cohésion entre les répliques devant se répondre. Bien que les répliques initiales de la confidente soient absentes, il y a bien l'illusion d'un enchaînement basé sur une réaction émotive. Les propos de l'allocutaire sont alors à imaginer, à reconstituer selon un principe de cohérence non descendante mais ascendante, pour remonter le fil d'une communication tronquée et non dite. Toutes les tournures exclamatives et interrogatives créent ainsi l'impression d'une situation dialoguale (de dialogue) avec un destinataire présent au moment de la scène d'énonciation pour qu'il y ait confidence, et pour que la confidence puisse être considérée comme un acte de langage. Un acte de langage (depuis Austin, 1962, et Searle 1969), c'est la construction d'une relation interactionnelle et interpersonnelle qui a besoin pour exister d'un émetteur et d'un récepteur, avec un message, et différentes fonctions langagières à activer pour que ce dernier soit encodé puis décodé. Pourtant, si l'acte d'écrire est un acte de langage, celui-ci pose traditionnellement un problème d'identification et de statut car il est monolocutif. Au sein de cette épineuse situation de monolocutivité d'écrivains devant imaginer leurs récepteurs, le théâtre et la poésie ont des fonctionnements spécifiques aux antipodes l'un de l'autre. Le théâtre est l'art de l'illusion de mettre en scène une suite finement imbriquée d'actes de parole hiérarchisés entre plusieurs actants, tandis que la poésie joue sur une scénographie énonciative de solitude et de souveraineté d'un Moi. Les dialogues d'une pièce de théâtre, en s'appuyant sur des marqueurs d'oralité, activent la fonction phatique définie par Jakobson en 1963, pour créer et maintenir la communication avec autrui. Celle-ci se fait beaucoup plus rare dans la poésie lyrique où même dire je t'aime, ou s'écrier hélàs, c'est mettre l'accent sur les émotions de l'énonciateur et jouer ainsi sur la fonction expressive/émotive du langage. L'on comprend alors l'aspect inédit que Marceline Desbordes-Valmore confère à ses élégies de confidence, entre monolocutivité et interlocutivité, avec l'enrichissement du schème expressif d'énonciation marqué cette fois par un phénomène d'inter-généricité, avec la co-présence de fonctions langagières dominantes dans chacun des deux genres littéraires.

(26) Dans cette présence d'un véritable style de l'oralité avec des marqueurs sémanticodiscursifs, un autre mimétisme est à l'œuvre, celui du souffle de la parole d'un personnage aux prises avec des émotions dans le feu de l'action :

$\mathrm{T} 1 / \mathrm{T} 2 / / \mathrm{T} 3 \mathrm{~T} 4$

J'ai couru... je suis faible... et ma langue glacée

$\mathrm{T} 1 \mathrm{~T} 2 \mathrm{~T} 3$

Peut à peine... Ma sœur, je l'aime donc toujours !

Les points de suspension et le rythme des vers concourent à la monstration d'un pathos avec un deuxième vers $(4 / 2 / / 6)$ qui casse la régularité rythmique attendue après le premier vers, un tétramètre $(3 / 3 / / 3 / 3)$, et confèrent l'impression d'une parole essoufflée, d'une parole soumise aux contraintes de l'oralité dont devaient impérativement tenir compte les dramaturges pour mimer un parlé plus naturel, et pour donner aussi le temps aux comédiens de respirer :

C'est ici... Pardonnez, je respire avec peine ;

mes genoux affaiblis me forcent à m'asseoir ${ }^{24}$, 
Avec cette mimétique de l'essoufflement, Marceline Desbordes-Valmore a élaboré ses vers avec des règles propres au genre théâtral, positionnant l'action au cœur de la métrique pour déployer une véritable dramaturgie, au sens étymologique du terme, c'est à dire un art de l'action, et plus précisément un art de la mise en situation d'une parole dans l'action.

\subsection{Du mouvement avant toute chose !}

50 (27) Cette dramaturgie place aussi l'action au cœur de la sémantique cette fois, avec un art de la mise en situation de personnages dans l'action. Le sémantisme passe par un recours très fréquent aux verbes d'action, déjà mentionné pour les scenarii :

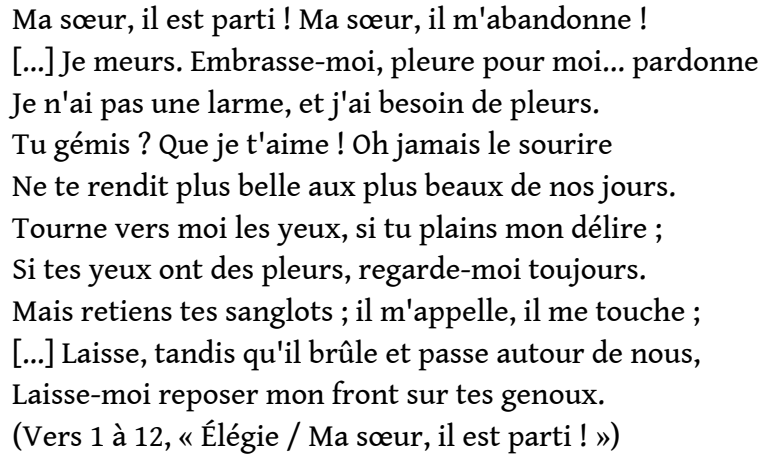

51 La confidente existe par ce qu'en dit le sujet : elle apparaît sous le prisme d'une désignation indirecte et ultra subjective avec les modalités. Plus encore, avec l'accumulation de verbes d'action, elle prend place et existe dans une relation de subordination au sujet lyrique qui en fait une figure passive : « embrasse ", « pleure ", « pardonne ", " retiens », « regarde ", « laisse "... Plus encore, ces verbes nous renvoient aussi aux actes de langage, avec une dimension locutoire, qui informe tant la confidente que la lectrice, et surtout une dimension illocutoire directive pour faire agir la confidente tout autant que la lectrice. La confidence valmorienne consiste donc à véhiculer et partager des états affectifs pour influer émotionnellement sur un lectorat dans une orientation pragmatique : « écoute » et " pleure ».

(28) Ces impératifs centrent l'acte de langage sur le destinataire et s'inscrivent alors dans la modalité allocutive, ou dans le système allocutif, " repérable à l'injonction, à l'interrogation, à l'interpellation, qui permettent de préciser le rapport entre locuteur et interlocuteur, et donc la manière dont ils sont impliqués dans l'acte de parole » (Détrie 2003 : 36). Ces trois marqueurs d' allocutivité sont présents et montrent ainsi la manière dont le sujet lyrique se positionne vis-à-vis des propos qu'il énonce et vis-à-vis de sa partenaire discursive (Charaudeau 1992) qu'il domine, qu'il dirige comme un metteur en scène tout puissant.

(29) Ces verbes d'action introduisent aussi la notion de geste et de corporalité, indispensables à l'écriture théâtrale car préfigurant la communication scénique non verbale. Et c'est tout un travail de suggestion d'une corporalité, et d'une corporalité toujours en mouvement, qui se donne à lire avec la nomination de différentes parties du corps qui s'ajoutent au sémantisme des verbes, que cela soit pour le destinataire ou pour le sujet lyrique, existant aussi par le mimétisme d'une respiration essoufflée, ajoutant à la représentativité corporelle. 
(30) Cette corporalité en mouvement et ces isotopies de l'action questionnent la nature du statut du Je et surtout du $\mathrm{Tu}$, entre personnages de théâtre et figures poétiques. La figure poétique serait passive, recevant des qualifications subjectives du sujet lyrique, demeurant muette et statufiée dans le hors temps d'une situation d'énonciation qui n'a pas la nécessité générique de créer une situation interactionnelle. La figure intéresse l'écrivain pour son potentiel représentatif et figuratif, d'où son appellation, devenant le symbole de tel ou tel concept. Pour le personnage de théâtre, malgré la multitude de points de vue et de théories à son sujet, il peut être défini par sa mise en situation et par sa mise en action (Uberfeld, 1977), puisqu' il agit et interagit avec les autres. Cela est rendu possible par la corporalité dont il est pourvu, avec le langage non-verbal, assurant un lien émotionnel et charnel entre le Je et le $\mathrm{Tu}$, « laisse-moi reposer mon front sur tes genoux ", décrivant une position qui aurait pu être mentionnée en didascalies dans un texte dramaturgique. Celle-ci permet à un comédien de l'interpréter et à un lecteur de le visualiser mentalement. Avec cette obsession du mouvement qui rend patente l'identité hybride de ces figures - personnages, la théâtralité acquiert un troisième sens, celui d'un espace conceptuel et discursif entre la scénographie littéraire et la représentation scénique.

\subsection{Dialogisme et intertextualité : le style de la tragédie}

(31) L'écriture valmorienne rend aussi la confidence théâtrale à travers l'emploi d'un certain lexique, de thèmes, de situations, voire aussi d'une métrique, qui rappellent grandement le théâtre classique, avec un syncrétisme dialogique opéré entre les différentes tragédies du 17ème siècle qu'elle connaissait, en particulier celles de Racine et de Corneille dont on retrouve les accents de la passion amoureuse :

Le revoir ! ô terreur ! l'entendre ! lui répondre !

Reconnaître ses yeux qui m'ont donné la mort ;

Les voir errer sur moi, sans trouble, sans remords !

Balbutier son nom, m'égarer, me confondre !

Desbordes-Valmore réutilise le motif de la fuite face à un impossible amour ou face à un amour perdu, ainsi qu'une obsession du regard et une esthétique cathartique et unificatrice des larmes à verser collectivement :

Non, laissez-moi m'enfuir. Que je doute de moi-même

Si je l'ai vu jamais, si j'existe, si je l'aime!

Ah, je ne le haïs pas, je ne sais point haïr :

Mais laissez-moi douter... Mais laissez-moi m'enfuir !25

Il semblerait même qu'elle se soit positionnée davantage du côté de Corneille, avec des coupes très marquées, un rythme haché pour faciliter la respiration des comédiens en mouvement, de nombreux verbes d'action, ainsi que des émotions paradoxales et des dilemmes, comme secourir un homme qui l'a fait souffrir. Plus encore, dans les vers précédemment cités, le lecteur pourra identifier une réécriture du vers le plus connu du Cid ${ }^{26}$ prononcé par Chimène, qu'elle interpréta d'ailleurs : " Ah ! Je ne le haïs pas ", rappelant le « Va, je ne te hais point ! ». Il y a réemploi du verbe haï, de la litote, d'une phonétique restant dans la gamme vocalique des [a], [ $\varepsilon$ ] et [э], avec un même nombre de pieds, un même rythme formé sur des mots tous monosyllabiques, portant les mêmes accents toniques et oratoires, etc. L'allusion dépasse donc les mots pour porter aussi sur la phonétique et sur la rythmique. Quant au « laissez-moi » en antépiphore dans « Le Retour chez Délie » ou le « laisse-moi » dans l' «Élégie /Ma sœur, il est parti », ils rappellent le « 
laisse-moi » que Chimène prononce à la fin de la même scène en s'adressant à Elvire, sa gouvernante... et confidente.

(32) L'écriture valmorienne témoigne donc d'une mémoire discursive (Moirand 2007) de la tragédie poussée jusqu'à la transgénéricité, avec des textes semblables à des monologues tragiques, jusqu'en leur volumétrie, avec152 vers pour « Le Retour chez Délie » et 161 vers pour « Révélation ». Pourtant un bémol est à émettre pour le cycle de Délie, dont le nom même maintient encore les écrits dans la revendication d'une certaine tradition poétique. Quant à l'intentionnalité ou non de cette intertextualité, DesbordesValmore raconta qu'elle écrivait entre deux actes. Que l'anecdote fut vraie ou non, elle montre que sa relation à l'écriture fut liée au théâtre, avec des vers qu'elle connaissait par cœur et dont il devait être difficile de se défaire. Les mécanismes mémoriels purent être activés inconsciemment ou/et consciemment, avec de possibles visées stratégiques : légitimer son arrivée sur la scène littéraire en jouant sur une sensibilité théâtrale pour être acceptée et encouragée par un public qui la connaissait déjà en tant que comédienne, appuyant son ethos discursif auctorial sur sa réputation, c'est à dire sur son ethos préalable. Cela pouvait aussi avoir l'utilité d'offrir des gages de respectabilité avec des vers estampillés d'un ton tragique, compatible avec celui de l'élégie qui pouvait être investie par une femme. Nous pouvons même élaborer encore une autre hypothèse à partir d'un constat, celui qu'il n'y avait pas ou peu de femmes s'illustrant dans la tragédie. Nous avons les exemples d'Olympe de Gouges ou de Constance Pipelet qui s'y essayèrent, mais le nombre des représentations demeura très limité, et elles furent taxées d'orgueil pour être sorties des écrits admis pour les femmes. Si la tragédie, et plus généralement les pièces de théâtre en lien avec la sphère publique, faisait partie des chasses gardées masculines, alors Marceline Desbordes-Valmore aurait pu sciemment s'adonner au style de l'écriture théâtrale en travestissant celle-ci sous les voiles d'un poème, c'est à dire en la déplaçant dans la sphère du poétique et de l'intime pour ne pas s'attirer les foudres des critiques, tout en parvenant à démontrer ses capacités.

\section{Conclusion}

59 (33) Le déploiement d'une confidence n'apparait explicitement que dans une petite vingtaine de poèmes, avec la figure d'une sœur, ou avec Délie, ou avec la mise en place d'isotopies, ce qui est peu au regard des centaines de poèmes composés par l'auteure. Pourtant cette confidence a aussi retenu l'attention de Victoire Babois qui la considéra comme un trait constitutif de la poésie de Desbordes-Valmore lors d'un hommage qu'elle lui rendit :

Valmore sait du cœur chercher tous les secrets

Et de ses vers touchants les aimables attraits

Pour mieux nous deviner se glissent dans notre âme

$[\ldots]$

Comme elle, nous croyons souffrir,

Et de son cœur sortis, ses soupirs, ses alarmes,

Portent l'émotion dans le fond de nos cœurs.

[...]

On écoute une mère, une amie, une amante,

Et le poète est oubliéén.

Ces vers réactivent à leur tour une molécule sémique de la confidence in absentia avec les sèmes du secret, de l'écoute et du partage émotionnel qui s'appliquent parfaitement à la 
confidence valmorienne, dont le travail sur une intimité théâtralisée permet à l'écriture d'une relation affective interpersonnelle de devenir un art suprême de l'illusion chez une comédienne-écrivaine et une écrivaine-comédienne. Et sa lectrice serait une spectatrice assise dans le fauteuil de son salon, avec des textes lui donnant la possibilité de visualiser aisément les scènes d'une confidence en mouvement qui pourraient se dérouler sous ses yeux, ou auxquelles elle pourrait être incluse, avec le sentiment d'être la destinataire privilégiée d'une écrivaine, avec une expansion empathique de la souffrance confiée.

61 (34) Concernant l'élaboration et les procédés sur lesquels repose la confidence, nous présentons un schéma qui en résume le fonctionnement riche et complexe.

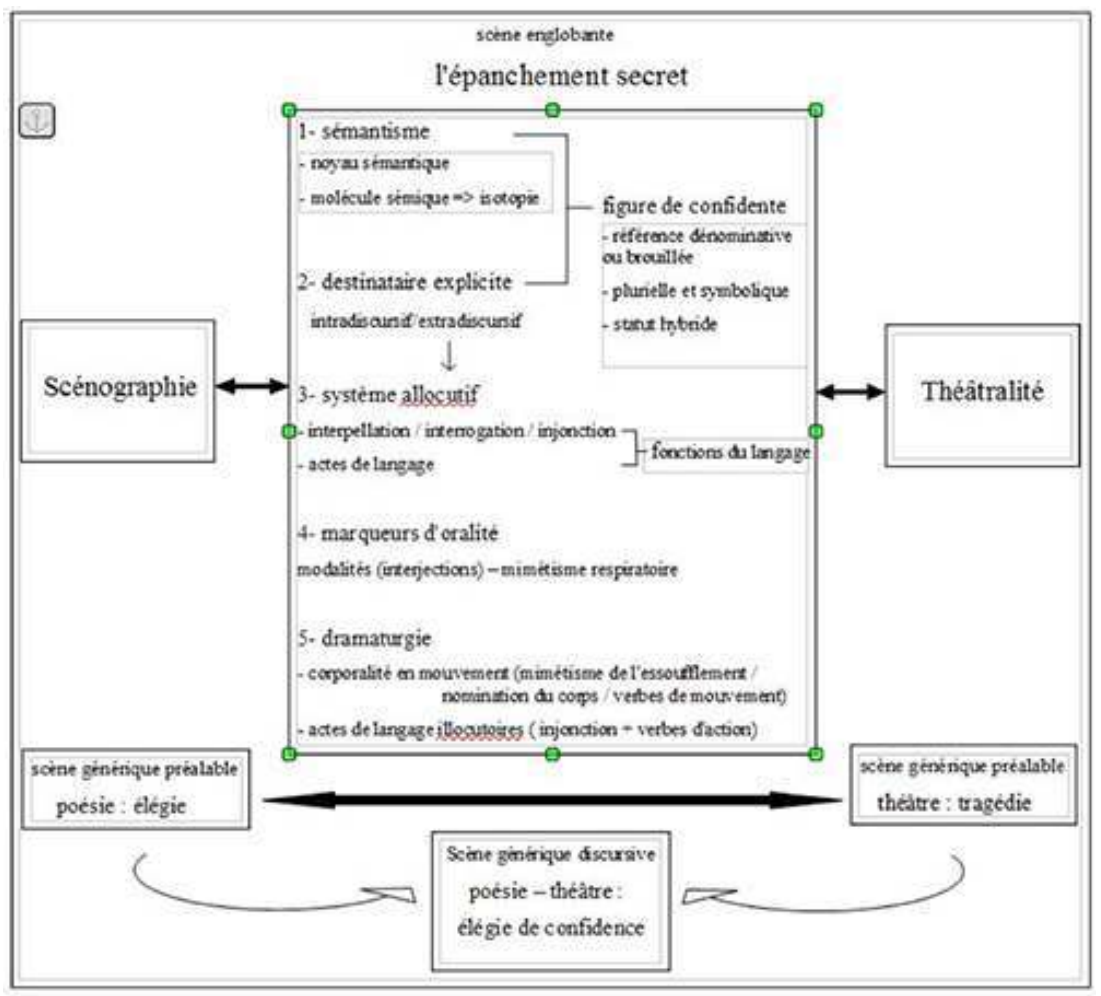

Figure 1. Schéma de la confidence implantés dans le genre poétique, considéré alors comme la scène générique initiale. Mais l'inverse aurait pu être vrai aussi. L'auteure aurait pu concevoir une scénographie littéraire de la confidence à partir du genre théâtral, en lui implantant des fonctions et des procédés figuratifs du genre poétique. Par ce schéma, avec un tableau pouvant se lire de droite à gauche et de gauche à droite, nous émettons une seconde hypothèse en complémentarité avec la première : celle d'un mouvement de va-et-vient permanent entre les deux genres et leurs spécificités, pour illustrer ce souffle de libre création repoussant les frontières génériques et permettant d'aboutir à ce qui pourrait être considéré comme un nouveau sous-genre élégiaque : l'élégie de confidence, se nourrissant de deux scènes génériques à considérer alors comme préalables.

(35) Dans la continuité d'André Chénier, de Victoire Babois et des théories de Germaine de Staël, Marceline Desbordes-Valmore a probablement occupé une place importante dans cette évolution du lyrisme par sa proposition originale d'une scénographie de la confidence. Elle permit de superposer les plans pour faire croire que c'était l'écrivaine qui 
se racontait, et non une instance lyrique artificielle et clairement dissociée et dissociable d'elle. Le motif de la confidence devint alors une procédure discursive produisant un effet de réel (Charaudeau, 1983 : 95). D'un point de vue formel, l'élaboration de poèmes monologues fit de l' hybridation générique un procédé dynamique offrant un renouveau dans l'écriture et ses artifices stylistico-discursifs, prenant place dans l'élaboration et la consolidation du romantisme qui aima à abolir les frontières entre les genres et les registres.

(36) Il est probable que ces élégies théâtrales eurent un impact sur les écrivains des générations suivantes qui se dirent lecteurs de Marceline Desbordes-Valmore. Alfred de Musset, par exemple, a nourri son art des deux mêmes genres en écrivant des recueils de poésie et des pièces de théâtre, et conçut son drame Lorenzaccio (1834) comme " un théâtre dans un fauteuil ", repensant, dans un mouvement inverse mais similaire en intention, le théâtre par rapport à la fonction émotive de la poésie, alors que Marceline Desbordes-Valmore avait repensé la poésie par rapport à la fonction phatique du théâtre. Pour Charles Baudelaire, il s'adressa à son lecteur par l'accroche « mon frère » dans Les Fleurs du Mal (1856), dans une relation interpersonnelle devenue un élément clé de la construction identitaire du sujet lyrique, réinterprétant ainsi sa relation au lecteur dans une sensibilité qui sembla vibrer en écho de celle qu'il surnommait " la grande Desbordes-Valmore ».

(37) Enfin, cette inventivité et cette hybridation générique, culminant dans ce que nous considérons pleinement comme des arts poétiques, nous font redécouvrir la dimension intellectuelle passée sous silence des écrits de cette femme de génie et de ce poète caméléon qui sut jouer subtilement des codes, tant scripturaux que sociétaux, pour pouvoir exister par l'écriture.

\section{BIBLIOGRAPHIE}

Amossy, Ruth. 1999 (dir.). Images de soi dans le discours. La construction de l'ethos (Lausanne : Delachaux et Niestlé)

Austin (John Langshaw), Quand dire, c'est faire, Paris, Seuil, 1970.

Authier-Revuz, Jacqueline. 1984. « Hétérogénéité(s) énonciative(s) », Langages 76, 98-111.

Authier-Revuz (Jacqueline), Ces mots qui ne vont pas de soi. Boucles réflexives et non-coïncidence du dire , Paris, Institut Pierre Larousse, 1996.

Baby, Hélène. 2005. «Introduction générale », Fiction narrative et hybridation générique dans la littérature française (Paris : L'Harmattan)

Brémond, Claude. 1973. La Logique du récit (Paris : Seuil)

Charaudeau, Patrick. 1983. Langage et discours. Eléments de sémiolinguistique (Théorie et pratique).

(Paris : Classiques Hachette)

Charaudeau, Patrick. 1992. Grammaire du sens et de l'expression (Paris : Hachette) 
Charaudeau, Patrick, \& Montes, Rosa. 2004 (dirs). La voix cachée du tiers (Paris : L'Harmattan)

Détrie, Catherine. 2003. «L'apostrophe dans Les Fleurs du mal. Stratégies textuelles et modalités de saturation de la place allocutive ", L'information grammaticale 96, 35-39

Dion, Robert, Fortier, Frances, Haghbaert, Elisabeth. 2001 (dirs.), Enjeux des genres dans les écritures contemporaines (Quebec : Nota Bene)

Galatanu, Olga. 1999. « Le phénomène sémantico-discursif de déconstruction-reconstruction des topoï dans une sémantique argumentative intégrée », Langue Française 123, 41-51

Garric, Nathalie, \& Calas, Frédéric. 2007. Introduction à la pragmatique (Paris : Hachette Supérieur) Jakobson, Roman. 1963. « Linguistique et poétique », Essais de linguistique générale (Paris : Minuit), 209-248.

Kerbrat-Orecchioni, Catherine. 2009 [1980]. L'énonciation. De la subjectivité dans le langage (Paris : Colin)

Lejeune, Philippe. 1975. Le pacte autobigraphique (Paris : Seuil)

Maillochon, Isabelle, \& Bassano, Dominique. 2003/2. « La compréhension des modalités déclaratives et exclamatives en français à 20 mois et 30 mois », Enfance 55 (Paris : PUF), 119-138 Mihajlovska, Lupka. 2012 . L'hybridation dans l'œuvre de Jeanette Winterson (thèse de doctorat en Langues et Littératures étrangères, Université d'orléans, soutenue le 16/11/2012)

Moirand, Sophie. 2003. « De la nomination au dialogisme : quelques questionnements autour de l'objet de discours et de la mémoire des mots ", Actes du colloque jeunes chercheurs Dialogisme de la Nomination (Montpellier : PULM)

Moirand, Sophie. 2007. " Discours, mémoire et contexte. Le rôle de l'allusion dans la presse », Corela, H-6, « Cognition, discours, contexte », Achard-Bayle, Guy \& Paveau, Marie-Anne (dir.)

Moirand, Sophie. 2007. Les discours de la presse quotidienne, observer, analyser, comprendre (Paris : PUF)

Naugrette, Catherine. 2010. L'esthétique théâtrale, 2e éd., (Paris : Armand Colin)

Planté, Christine. 2015 [1989]. La petite sœur de Balzac. Essai sur la femme auteur (Lyon : PUL)

Rastier, François. 1987. Sémantique interprétative (Paris : PUF)

Rochette, Bruno. 2007. « Eléments hors structure dans la Cena Trimachionis », Bodelot, Colette (éd.), Eléments « asyntaxiques » ou hors structure dans l'énoncé latin (Clermont-Ferrand : Presses Universitaires Blaise Pascal), 265-294

Rosier, Laurence. 2004. «Interjection, subjectivité, expressivité et discours rapporté à l'écrit : petits effets d'un petit discours ", Cahier de praxématique 34, 19-49.

Rosier, Laurence. 2006. « De la vive voix à l'écriture vive. L'interjection et les nouveaux modes d'organisation textuels », Langages 161 (Paris : Colin/Dunod), 112-126.

Searle, John. 1972. Les actes de langage : essai de philosophie linguistique ( Paris : Hermann)

Sermet, Joëlle de. 1996. «L'adresse lyrique », Figures du sujet lyrique, Rabaté, Dominique (dir.)

(Paris : PUF), 81-97

Siblot, Paul. 1997. « Nomination et production de sens : le praxème ", Langages 127, 39-55

Siblot, Paul. 1998. «Signifiance du praxème nominal », L'information grammaticale 77, 24-27

Stolz, Claire. 2006. Introduction à la stylistique (Paris : ellipses) 
Vratskidou, Eleonora. 2011. « Connaître, sentir, agir : le génie chez Johann Gottfried Herder », Kremer, Nathalie (ed.), Le génie créateur à l'aube de la modernité (1750-1850), (Lille : Septentrion), 41-57

Ubersfeld, Anne. 1977. Lire le théâtre (Paris : Belin)

\section{NOTES}

1. Des remerciements aussi à Madame Françoise Nicol.

2. Définition donnée par Le Petit Larousse.

3. Quatre dictionnaires ont été utilisés pour cette étude sémantique : Le Petit Larousse, le Trésor de la Langue Française, le Larousse en ligne et l'Internaute.

4. Les derniers 8 vers de "Révélation ", Les Pleurs, 1833.

5. Le sémème de la communication peut être décomposé en unités plus petites que sont les sèmes, c'est à dire des traits sémantiques pertinents et indispensables entrant dans la définition de la communication.

6. Vers 1 du poème « Je l'ai vu».

7. Liste des poèmes constituant le corpus d'étude autour de la figure de la sœur : «A ma sœur / Que veux-tu ? Je l'aimais », « A ma sœur / Qu'ai-je appris ! Le sais-tu ? », « Élégie / Ma sœur, il est parti », « Élégie / Quoi ! Les flots sont calmés », « Je l'ai vu », « A Madame Amable Tastu », une autre poétesse, «A celles qui pleurent », « La Mémoire »...

8. «A ma sœur / Que veux-tu ? Je l'aimais », vers 44, dans Poésies de 1830, in CEuvres poétiques de Marceline Desbordes-Valmore, édition complète établie et commentée par M. Bertrand, Presses Universitaires de Grenoble, 2 vol., 1973, p. 78.

9. Le pronom " vous " n'est employé que dans un seul poème, celui dédié à la poétesse Amable Tastu. L'on y retrouve le syntagme " ma sœur », et une scénographie de la confidence des cœurs entre deux femmes écrivaines.

10. Liste de poèmes s'ajoutant à notre corpus : « Délie I ", « Délie II ", « Délie III », « Le Retour chez Délie ».

11. «Le retour chez Délie », v.112, 119, 141,144, dans Poésies de 1830, op. cit., p. 161 à 164.

12. Célèbre poète de la Renaissance, contemporain et ami de Louise Labé. voire le véritable auteur des poésies de Louise Labé pour ceux qui soutiennent la théorie de la supercherie littéraire.

13. «A Ma sœur / Qu'ai-je appris !...», v. 1, 3 et 4, dans Poésies de 1830, op. cit., p. 80.

14. «Élégie / Quoi ! Les flots sont calmés », vers 1 à 3, dans Poésies de 1830, op. cit., p. 67.

15. Confondu aujourd'hui à tord avec l'accent tonique, l'accent oratoire servait à exciter les émotions et correspondait au marquage vocal le plus fort pour l'orateur. Il était pourvu d'une fonction pathétique, d'où l'autre nom donné à cet accent. La présence d'un accent oratoire confirme l'importance de l'oralité/oralisation théâtrale du vers chez Desbordes-Valmore.

16. Madame de Staël-Holstein, De l'Allemagne, dans CEuvres complètes de Madame la Baronne de StaëlHolstein, Tome II, Paris, Firmin Didot Frères et Treuttel et Würtz Librairies, 1844, p. 60.

17. Vers 157, « Révélation ».

18. Vers 93, op. cit., p. 199.

19. Théorie complexe sur l'art, d'origine religieuse, dont on trouve trace dans la peinture du Quattrocento à Florence. La théorie du speculum mundi postulait que l'art était un miroir du monde (et non le monde en lui-même), en établissant un jeu de correspondance entre le sensible et l'intellectuel, entre l'humain et le divin à célébrer. L'idée essentielle était que l'œuvre d'art comme production humaine n'était qu'une imitation, qu'une reproduction, toujours inférieure à une nature à la beauté supérieure et inégalable car créée par Dieu. La théorie perdit 
progressivement sa provenance religieuse à partir de la Renaissance pour ne conserver que le concept de réflexivité. Au début du XXe siècle, Proust défendit l'idée adverse, celle qu'une œuvre participe au monde en s'intégrant dans la matérialité de la réalité.

20. Vers 2, 5-8, 19-20, op. cit., p. 444.

21. Nous entendons le terme ethos au sens d'image que l'on cherche à donner de soi et que l'allocutaire co-construit par des interprétations. Cf. Images de soi dans le discours. La construction de l'ethos, sous la dir. d'Amossy.

22. Nous renvoyons à l'ouvrage réédité de Christine Planté sur la femme artiste au $19 \mathrm{e}$ siècle : La petite sœur de Balzac. Essai sur la femme auteur.

23. Excepté « hélàs » et « ô », interjections codifiées pour les genres littéraires nobles, devenues des préconstruits abondamment utilisés dans la tragédie et l'élégie.

24. « Le Retour chez Délie », v. 1-2.

25. « Le Retour chez Délie ", dernier quatrain.

26. L'Acte III, scène 4.

27. Victoire Babois, "Épître à Clotilde de Surville. 1827 ", dans le recueil Epîtres, paru dans Elégies et poésies diverses de Madame Babois, dans Euvres, tome I, troisième éd., Paris, Nepveu, 1828, p. 205-206.

\section{RÉSUMÉS}

Cet article se donne deux objectifs l'un au regard de l'autre : étudier le cas d'une écriture littéraire hybride, entre poésie et théâtre, et étudier le motif de la confidence qui parcourt certaines élégies de la poétesse Marceline Desbordes-Valmore. A l'image de ce double titre, entre champ linguistique et champ littéraire, il s'agira d'articuler ces deux domaines dans une perspective interdisciplinaire. La confidence sera abordée sous l'angle des théories stylistiques, sémantiques et pragmatico-énonciatives à unifier entre elles pour se réapproprier l'Analyse du discours littéraire, désormais ADL (Maingueneau, Amossy, Adam, Rastier, etc), et pour comprendre comment des éléments et procédés dramaturgiques implantés dans des poèmes confèrent une théâtralité à ceux-ci, théâtralité qui contribue à un renouveau du genre élégiaque à une période charnière de la poésie française.

this article aims both at studying the case of a hybrid literary writing between poetry and drama and at studying the pattern of confidence which can be found in some elegies by the poetess Marceline Desbordes-Valmore. Like this double title, between linguistic field and literary field, it is about linking together these two fields from an inter-disciplinary viewpoint. Confidence will be dealt with from stylistic, semantic and pragmatic-enunciative viewpoints that must be united in order to appropriate again the analysis of literary discourse, henceforth referred to as ALD (Maingueneau, Amossy, Adam, Rastier, etc) and to understand how elements and drama processes introduced in some poems endow them with theatricality, a theatricality which contributes to the renewal of the elegiac genre at a turning point of French poetry. 
INDEX

Mots-clés : Marceline Desbordes-Valmore, Analyse du discours, hybridation générique, figure lyrique, énonciation, dialogisme interdiscursif.

Keywords : discourse analysis, generic hybridization, figure lyric, enunciation, dialogism interdiscursive.

\section{AUTEUR}

\section{ELENA THUAULT}

CoDiRé

Université de Nantes

$<$ elena.thuault@gmail.com> 